José J. González Millán javier.gonzalezmillan@uptc.edu.co Calle 17 No 11a-16

Sogamoso, Boyaca - Colombia.

Magister en Administración Universidad Nacional de Colombia. Profesor Asistente Escuela de Administración de Empresas de la Facultad Sede Sogamoso de la Universidad Pedagógica y Tecnológica de Colombia. Director Centro de Investigación y Formación Avanzada - CIFAS. Coordinador grupo de investigación Management.

Miryam T. Rodríguez Díaz miryamteresar492@gmail.com Calle 22 No 11b-03

Sogamoso, Boyaca - Colombia.

Especialista en Gestión del Talento Humano de la Universidad Pedagógica y Tecnológica de Colombia. Estudiante Maestría en Administración Universidad Nacional de Colombia. Docente Auxiliar Escuela de Administración de Empresas UPTC. Investigadora grupo de investigación Management.

Liliana M. Duarte mojarrada@hotmail.com Calle 17 No 11a-16 Sogamoso, Boyaca - Colombia.

Administradora de Empresas Universidad Pedagógica y Tecnológica de Colombia. Investigadora grupo de investigación Management.

Artículo de investigación científica y tecnológica Según Clasificación Colciencias

Fecha de recepción: agosto 9 de 2011 Fecha de corrección: diciembre 13 de 2011 Fecha de aprobación: diciembre 20 de 2011

\section{Necesidades de consultoría en las MIPYMES del Valle de Sugamuxi en Boyacá}

\author{
Needs for consultancy in micro and SMES in Valle de Sugamuxi \\ in Boyacá - Colombia
}

Besoins de consultation dans les petites et moyennes
entreprises (PME) de la Vallée du Sugamuxi à Boyaca 
Needs for consultancy in micro and SMES in Valle de Sugamuxi in Boyacá - Colombia

Necesidades de consultoría en las MIPYMES del Valle de Sugamuxi en Boyacá

Besoins de consultation dans les petites et moyennes entreprises (PME) de la Vallée du Sugamuxi à Boyaca

\section{Abstract}

This article gathers the most relevant elements of the research titled Estudio de necesidades de Consultoría empresarial en el valle de Sugamuxi, en el departamento de Boyacá, conducted by the Management research group at UPTC, which applied 354 surveys to managers of the companies (Micro and SMEs) belonging to the region. The study determined the current situation of the companies, object of study, on the needs for business consultancy, the diagnosis of the differences between advisory and consultancy, identification of areas and neuralgic topics of this service, identification of the most relevant qualitative and quantitative factors for the creation of consultancy centers.

Keywords: consultancy, advisory, companies, development, productivity, business.
Besoins de consultation dans les petites et moyennes entreprises (PME) de la Vallée du Sugamuxi à Boyaca

\author{
Necesidades de consultoría en las \\ MIPYMES del Valle de Sugamuxi en \\ Boyacá
}

\section{Needs for consultancy in micro and SMES in Valle de Sugamuxi in Boyacá - Colombia}

\section{Résumée}

Cet article reprend les éléments les plus pertinents de la recherche intitulée "Étude des besoins de consultation organisationnelle dans la vallée du Sugamuxi, dans le département de Boyacá", développée par le groupe de recherche de Management de I' UPTC (Université Pédagogique et Technologique de la Colombie). On a enquêté pour cette recherche 354 directeurs des PME de la région.

L'étude a présenté la situation actuelle de ces entreprises : les besoins de consultation organisationnelle, le diagnostic des différences entre conseil et consultation, l'identification des sujets et des domaines névralgiques du service, l’identification des facteurs qualitatifs et quantitatifs les plus critiques pour la création de centres de consultation.

Mots clef: consultation, conseil, entreprises, développement, productivité, affaires. 


\section{Necesidades de consultoría en las MIPYMES del Valle de Sugamuxi en Boyacá}

\section{Introducción}

La consultoría para las organizaciones, hoy adquiere el carácter de práctica social altamente generalizada y aprobada por parte de los actores involucrados en las diversas actividades económicas, sociales, empresariales y organizacionales, constituyendo así, una excelente herramienta de gestión para las organizaciones, ya que al tiempo que soluciona problemas específicos en áreas definidas, brinda la posibilidad de resolver problemas organizacionales y administrativos, sin necesidad de asumir costos onerosos carentes de estudios, por tanto son, en la mayoría de las veces, no sostenibles, luego no se justifica su mantenimiento permanente en los rubros específicos de la organización. (Marc, y Stuart, 1997)

Respecto con lo dicho anteriormente, las empresas necesitan de personas capacitadas y conocedoras de temáticas empresariales que conozcan el mercado para ejercer la función consultora (Hompanera, 2002), de tal manera que los profesionales que presten sus servicios cumplan con características mínimas que permitan atender las necesidades del mercado en el cual se desempeñan. (Calegari y Karyna, 2001, p. 149)

En este artículo se manifiestan las necesidades de consultoría de los 354 gerentes de las Mipymes de la provincia de Sugamuxi en el Departamento de Boyacá, para tal fin, se estructura en cuatro capítulos principales. El primero versa sobre las generalidades y diferentes fundamentos teóricos; un segundo acápite recoge los elementos metodológicos más importantes tenidos en cuenta en la investigación; el tercer apartado trata la discusión de los resultados encontrados en el estudio y el cuarto se refiere a las conclusiones generales del estudio, terminando con los respectivos referentes bibliográficos.

\subsection{Fundamentos teóricos}

En el contexto histórico, Calderón y Castaño $(2005$, p. 762) parten desde la revolución industrial, con la aparición de la fábrica moderna y las transformaciones institucionales y sociales, dando origen a un estado en el cual la empresa hizo posible un proceso de generación y estructuración de experiencia gerencial relativamente avanzada, fue entonces cuando se hizo importante determinar y escribir los métodos y los principios aplicables a diversas organizaciones y situaciones, por lo cual los empresarios fueron inducidos y presionados para buscar una forma práctica para dirigir y controlar la empresa.

Frederick Taylor (1911) propone la Organización Científica del Trabajo, a través de los criterios técnicos y metodológicos básicos para simplificar los procedimientos de trabajo y aumentar la producción en la fábrica, principalmente en campos como productividad y eficiencia en las fábricas y talleres. (Calderón y Castaño, 2005, p. 762)

La posguerra permitió la rápida expansión de las empresas, gracias a la aceleración de los cambios tecnológicos, la aparición de nuevas economías en desarrollo y la intensificación de la internacionalización de las industrias, el comercio y las finanzas mundiales. Lo anterior trajo una serie de oportunidades particularmente favorables para la demanda de servicios de consultoría de empresas. (Calderón y Castaño, 2005, p. 762). La mayor parte de las organizaciones de consultoría que existen hoy se debe al poder y la reputación técnica lograda a través del tiempo. Por ejemplo PA, la empresa de consultoría más importante del Reino Unido, tenía sólo 6 consultores en 1943 , 370 en 1963; más de 1300 , dispersos por 22 países en 1984, y 1700 en 1993. A fines del decenio de 1980, en los Estados Unidos, se calculaba que el número total de consultores de empresas de dedicación plena era 100.000 veces mayor que el que existía a mediados de 1960. Todo esto genera una serie de cambios cualitativos importantes, tal como lo expresa Kubr (1986, p.33), a saber:

* Diversificación y ofrecimiento de servicios para satisfacer la necesidad de los clientes. 
* Políticas innovadoras mediante procesos técnicos en aspectos de gestión que puedan interesar a los clientes.

* Aumento en la competencia relacionada con la consultoría.

* Internacionalización constante en las grandes y medianas empresas mediante la difusión de actividades en busca de nuevos mercados.

* Consultoría interna empresarial mediante diagnósticos con profesionales empresariales.

* El empleo de consultores en organizaciones, privadas y públicas ha permitido aumentar la competitividad, tanto a nivel empresarial, como de clientes. Al respecto, existen básicamente tres perspectivas que identifican la consultoría como: un servicio, un proceso y un enfoque. Es de aclarar que puede existir una cuarta postura que tiene que ver con las asociaciones e instituciones propias del tema, tal como se analiza a continuación:

En primer lugar en lo concerniente a la definición de consultoría, autores como Andrade (2007) y Kubr (1986) la consideran como un servicio que tiene por función auxiliar a la organización en el análisis y solución de problemas de la vida práctica, los cuales aplican a diversos sectores de la economía. Bustelo y García (2000, p. 6) expresan que "la consultoría es un servicio de apoyo a las empresas para diseñar políticas de gestión de la información, definir estrategias para su implantación, proponer soluciones - actuaciones concretas en la materia" Ahora bien, Greiner y Metzger (1983, p. 6) consideran este proceso como "un servicio profesional de ayuda al desenvolvimiento de las organizaciones, por personas entrenadas y cualificadas que asisten, en forma objetiva e independiente, a una organización o cliente con el objetivo de identificar y analizar problemas administrativos, recomendar soluciones, y ayudar cuando sea solicitado la implantación de las mismas"

Igualmente, Orlickas (1998) y Block (1991) presentan una visión basada en el fortalecimiento de determinada prestación de servicio, mediante un profesional cali- ficado, conocedor de un tema, obviamente, bajo una remuneración de un proyecto para un determinado cliente. También para Larry Greiner y Robert Metzger (1983, p.16), la consultoría es un servicio de asesoramiento contratado y proporcionado por personas especialmente capacitadas y calificadas que prestan asistencia, de manera objetiva e independiente, a organizaciones, mediante tareas como detección de problemas de gestión, análisis, recomendaciones y soluciones a los mismos.

López y otros (2010, p. 152) afirman que la consultoría "es un servicio prestado por un profesional idóneo (con conocimiento, capacidad y experiencia) a una organización, con el fin de contribuir a la solución de sus problemas y el logro de sus objetivos". Este concepto tiende a identificar a la consultoría como un servicio prestado por una persona con amplio conocimiento, criterio y capacidad para tomar decisiones basada, en la experiencia adquirida.

En segundo lugar se encuentra la perspectiva de proceso en la consultoría. Para Schein, citado por Becerra (2002, p. 16) la consultoría es un ciclo de ayuda organizacional, en donde existe la relación cliente-consultor; en esta misma línea Miranda y Vitta (2008, p. 5) la definen como "el conjunto de actividades que realiza el consultor para ayudar al cliente a percibir y comprender los acontecimientos de los procesos que se presentan en el ambiente y a influir sobre ellos".

Viadu y otros (2006) la conceptúan como una secuencia para proporcionar ayuda sobre el contenido, el proceso y la estructura de tareas en que el consultor cumple, únicamente la labor de asesor. Milan Kubr (1994) la considera como método de mejoramiento de las prácticas de gestión; además, dilucida lo que es un proceso de consultoría en relación con la profesionalización de la actividad misma. Para el profesor Antornosi (2010, p.48) es un "proceso que proporciona ayuda sobre contenidos, procesos o estructuras cuando la persona que la ofrece no es efectivamente responsable de la ejecución de la tarea, sino que colabora con quienes lo son".

En tercer lugar aparece la perspectiva de la consultoría basada en el consultor; tiene la tarea de identificar la función del sujeto 
de la consultoría antes que el objeto mismo; al respecto, Parreira (1997, p.12) expresa el concepto en términos de proporcionar, dar, solicitar y pedir conceptos, opiniones, estudios de un experto para que auxilie y oriente un trabajo administrativo; a su vez Edna Rodríguez (2002) la considera como la contratación de un profesional, promotor de cambios, dado que las soluciones deben llegar mediante procesos de percepción de una realidad en términos de conocimiento y especialización propias de expertos, que para este caso es el consultor, es por este motivo que Lozano (2009, p. 216), manifiesta que "la consultoría es un hecho que requiere de una gran habilidad en el manejo de aspectos específicos reconocidos por los expertos".

También De Padua y Duarte (2005) afirman que el quehacer del consultor es mostrar a la empresa como aquel lugar en donde los miembros necesitan subsanar dificultades, pasando del discurso a prácticas efectivas; por tanto, los gerentes deberán demostrar su liderazgo para resolver problemas de la compañía en términos de una buena gestión empresarial.

Por último está la perspectiva de la consultoría a nivel de organizaciones. En este apartado se encuentran, en primer lugar, el Instituto de consultores de empresas en México, que define la consultoría de empresas como:

El servicio prestado por personas independientes y calificadas en la identificación e investigación de problemas relacionados con políticas, organización, procedimientos y métodos de trabajo de una organización y la recomendación de medidas apropiadas para su solución; y la prestación de asistencia en la aplicación de dichas recomendaciones. (ICEM, 2010, P.1)

Ahora bien, el Instituto Brasilero de consultoría (IBCO) identifica la consultoría como un proceso interactivo en el cual actúan un agente de cambios, ya sean internos o externos y un cliente, para lo cual un consultor asume las responsabilidades de auxiliar y colaborar a los ejecutivos en la toma de decisiones, sin tener control directo de las situaciones que van a ser cambiadas.
El licenciado Jhovan Castillo del Instituto Tecnológico Superior de Tamazunchale, al respecto, la concibe como:

La forma de proporcionar ayuda sobre el contenido, proceso o estructura de una tarea o de un conjunto de tareas, en que el consultor no es efectivamente responsable de la ejecución de la tarea misma, sino que ayuda a los que lo son.

El Instituto de Consultores de Empresas del Reino Unido, citado por Duarte y Fernández (2006, p. 28) define como "aquel servicio prestado por una persona o personas independientes y calificadas en la identificación e investigación de problemas relacionados con políticas, organización, procedimientos y métodos; recomendación de medidas apropiadas y prestación de asistencia en la aplicación de dichas recomendaciones". De tal manera que dichos servicios puedan ser presentados por empresas, profesionales autónomos, órganos internos, tanto públicos como privados; es decir, por personas idóneas y capacitadas en una rama o disciplina. (Kubr, 2007)

Por último, Schein (1988), citado por Miranda y Vitta (2008, p.4) propone un tipo de consultoría, basada en prácticas de orden interdisciplinar con pertinencia relativa en torno a una profesión o área del conocimiento en donde se encuentren administradores, ingenieros, licenciados, comunicadores, psicólogos, entre otros.

Para terminar, en cuanto a las diferentes posturas epistemológicas referidas con el tema de investigación, es prioritario afirmar que la consultoría es un proceso que permite a las empresas solucionar sus problemas mediante entidades externas. Donadone (2003) y Jacintho (2004); sin embargo, no siempre se presenta este recetario de mejora empresarial, por lo tanto, este servicio puede generar desconfianza en las organizaciones $y$ en los consultores. Entonces, el profesional de esta disciplina debe poseer fuentes de información adecuadas que permitan construir conocimiento y comprender los comportamientos empresariales, basados en una toma de decisiones serias y reales, demostrando la idoneidad del consultor. (Mafra y Rodríguez, 2007) 


\section{Actitud}

* Conoce las dinámicas que se manejan en la empresa en particular

* Su conocimiento se concentra en las estrategias de la empresa en que se encuentra

* Se alinea a lo que el cliente quiere hacer

* Poco conocimiento de área de negocios, se limita a los procesos

* Su intervención es espontánea

* Supervisa y ejecuta al mismo tiempo

* Juventud

* Superficialidad

* Su manejo se basa en "teorías"

* Herramientas: puede ser mas creativo

* Utiliza medios publicitarios para anunciar sus servicios

* Entrega el trabajo en fechas
Actitud

* Conoce las dinámicas que interactúan en el mercado

* Conoce las estrategias del grupo empresarial por entero.

* Es creativo en la dinámica del cliente.

* Involucrado con el medio a través de la lectura de temas de actualidad: el cliente espera que sepa de negocios.

* Crea metodología de intervención con el cliente

* No supervisa lo que no ha ejecutado

* Experiencia

* Profundidad

* Innovación en los negocios

* Herramientas: se puede enlatar

* Sus servicios son conocidos por medio de referencias en el medio en el que se mueve

*Agrega valor económico a su trabajo, eficiencia y eficacia

\section{Fuente: Duarte y Fernández, 2006, p. 31, adaptado de www.pyme.com.mx/administracion/index.php}

Donina (2009), basado en Fowler (2005), describe dos tipos de consultores. Los de más experiencia, los llamados senior y los más jóvenes, los denominados junior. Para complementar dicho aspecto Duarte y Fernández (2006), presentan un comparativo de las principales actitudes de los consultores Junior y Senior. (Cuadro 1).

En lo referente a los múltiples tipos de consultoría, estudiosos como Pérez (2006) la clasifican fundamentalmente bajo dos enfoques, uno centrado en los negocios (organizacional o empresarial) y otro basado en el valor. El segundo caso ha sido poco explorado; sin embargo, su importancia radica en que incluye a los distribuidores, quienes van adquiriendo experiencia y, a la vez, cumpliendo un importante factor diferencial, hecho que constituye una ventaja competitiva. Al respecto, Neves y Campello (2008), consideran que es la consultoría interna la que mejores beneficios le genera a la organización, pues ellos son los verdaderos conocedores y artífices del conocimiento empresarial interno.

Respecto con la eficiencia y la eficacia, Piovezan $(2008$, p. 2) presenta el ciclo de los conceptos resumidos (Gráfico 1). Es importante aclarar que la eficiencia se relaciona con aspectos internos de la compañía, mediante la utilización de recursos, en tanto que la eficacia se ocupa de las aplicaciones de la consultoría con los distintos resultados de la empresa y sus posibles impactos en su estructura y operaciones propias de la misma (Gráfico 1).
Gráfico 1. Conceptos de Eficiencia y Eficacia de la consultoría

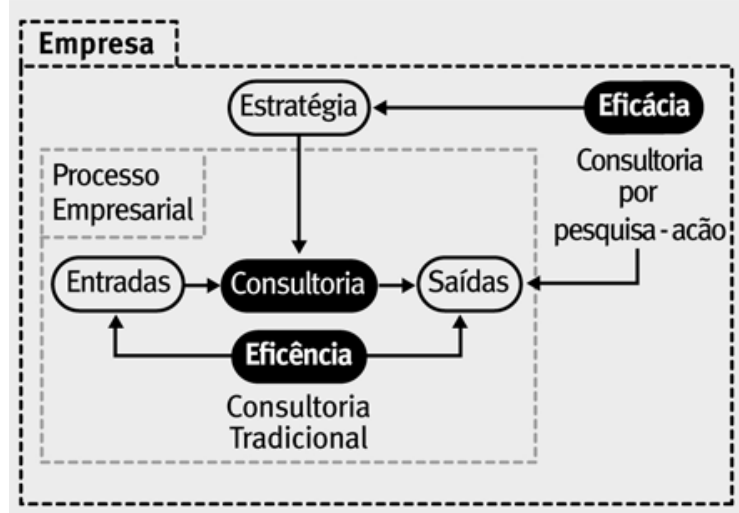

Fuente: Piovezan, 2008, p.2.

Al aludir a las distintas características de la consultoría y los consultores, es de resaltar el hecho de que en empresas importantes, como IBM, hacen uso de sus mejores prácticas corporativas mediante la armonización de las necesidades y los requerimientos de los clientes a fin de prestar servicios integrales a otras empresas (Velásquez y Vernaza, 2006). Calderón y Castaño (2005, p. 762) afirman que "bajo este panorama surgen hoy, grandes empresas consultoras como Booz Allel y Hamilton, A.D. Little, Mckinsy Consulting, y Andersen Consulting, entre otras, encargadas de expandir la práctica de la consultoría en Norte América y luego llevarla a Europa”. Con la conformación de estas grandes firmas norteamericanas creadas para asesorar y ayudar a la industria americana se da el origen al término consultoría.

Como complemento a los anteriores conceptos, Gerusa Rodríguez (2002) presenta las diferentes competencias y habilidades que constituyen el 
perfil de un consultor; estereotipo que obedece a una serie de dimensiones generales, dentro de las cuales se encuentran la Técnica, la Administrativa y la Psicosocial (gráfico 2).

Gráfico 2. Competencias y Habilidades de un perfil técnico de un consultor

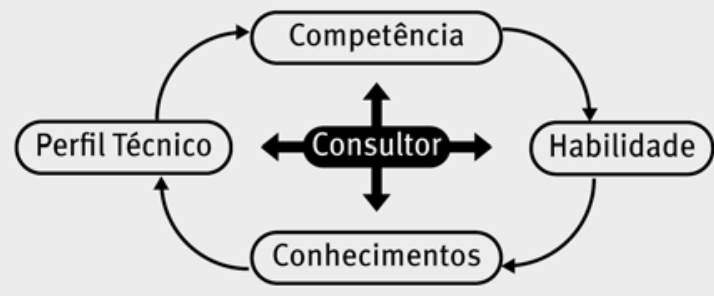

fuente: Gerusa Rodriguez, 2002, p. 20.

El área Técnica alude al conocimiento específico que se tiene del trabajo, mediante habilidades y técnicas propiamente dichas; la dimensión Administrativa se refiere al desarrollo de nuevas estrategias donde se pueda llevar a cabo la aplicación práctica del conocimiento teórico, y la faceta Psicosocial tiene que ver principalmente con las habilidades del consultor en términos de creatividad, motivación, equilibrio y percepción de la realidad, lo que conlleva en el proceso de consultoría a poder controlar percepciones, mitos, leyendas, creencias e ideologías muy propias de cada cultura organizacional consultoriada.

\section{Metodológia}

\subsection{Tipo y método de investigación}

Para alcanzar el objetivo propuesto en la investigación, fue necesario acudir a un estudio de carácter descriptivo-explicativo de acuerdo con Méndez (1998). El estudio permitió realizar una interpretación de las variables, objeto de investigación, mediante una sistematización y análisis de la información, tarea que condujo a una mejor descripción de las diferentes características propuestas en el estudio investigativo. (Camacho, 2003)

\subsection{Fuente y técnica de recolección de información}

Como fuentes primarias y de acuerdo con Tamayo (2002) se tuvo en cuenta a los Gerentes de las de las 354 Mipymes, así como a los funcionarios de la Cámara de Comercio de la ciudad de Sogamoso. Para la recolección de información, se utilizó como técnica la encuesta estructurada.

Para las fuentes secundarias se acudió a la legislación sobre creación de empresas, datos suministrados por la Cámara de Comercio de Sogamoso sobre el comportamiento histórico de la creación de empresas, diferentes normas y políticas. Además, se realizó la consulta de material documental.

\subsection{Población, muestra, tipo de muestreo y tratamiento de la información}

La población, objeto de investigación está conformada por 4.719 empresas distribuidas por tamaño (Cuadro 2)

\section{Cuadro 2. Clase y Cantidad de Empresas en la Provincia de Sugamuxi*}

\section{Cod. Actividad económica}

agricultura, ganadería, caza y silvicultura

pesca

explotación de minas y canteras

industrias manufactureras

suministro de electricidad, gas y agua

construcción

comercio al por mayor y al por menor

restaurantes y hoteles

transporte, almacenamiento y comunicaciones

intermediacion financiera

actividades inmobiliarias, empresariales y de almacenamiento

educación

servicios sociales y de salud

otras actividades de serv, comunitarios, sociales y personales

\begin{tabular}{|l|c|c|c|}
\hline & \multicolumn{3}{|c|}{ Tamaño de la empresa } \\
\hline \multirow{4}{*}{ Micro } & Peq & Med \\
\hline & 40 & 1 & 1 \\
\hline & 3 & & \\
\hline & 75 & 2 & \\
\hline & 699 & 9 & 5 \\
\hline & 2 & & \\
\hline & 144 & 7 & \\
\hline & 1.442 & 26 & 3 \\
\hline & 755 & & \\
\hline & 348 & 5 & 3 \\
\hline & 81 & 1 & \\
\hline & 419 & 5 & \\
\hline Total de empresas & 38 & & \\
\hline & 122 & 5 & 2 \\
\hline
\end{tabular}

$4 \cdot 719$ 
En el diseño de la muestra se tuvo en cuenta las siguientes variables: $N=4719$ empresas, $E=5 \%, Z=95 \%, P=52 \%$ y $Q=48 \%$, para conocer el tamaño de la muestra se utilizó la fórmula de poblaciones finitas que dio como resultado 354 empresas para ser encuestadas. El Cuadro 3 se presenta la ficha técnica que resume los elementos relevantes del diseño muestral.

\section{Cuadro 3. Ficha Técnica Encuesta}

Fecha de realización encuesta: Agosto y Septiembre 2010 Población: 354 Mipymes

Dirigida a: Gerentes y/o Administradores de las empresas.

Ciudad: Sogamoso.

Metodología: Encuesta. Cuestionario Escrito.

Realizador del estudio: Investigadores del proyecto.

Nivel de Confianza Obtenido: $95 \%$

Margen de error: $5 \%$

\section{Fuente: elaboración propia.}

Debido al tamaño de muestra, fue necesario clasificarlas básicamente en micro, pequeñas y medianas empresas, las que se establecieron porcentualmente, conservando su proporción real de la siguiente manera: 275 microempresas que equivalen al $78 \% ; 65$ pequeñas empresas al $18 \%$ y 14 medianas empresas al $4 \%$. El tipo de muestreo aplicado fue aleatorio simple, dando la misma posibilidad para todos los elementos muestrales; para el tratamiento de la información, la herramienta utilizada fue el paquete SPSS versión 20, complementado con el MS. Excel.

\section{Resultados y discusión}

A continuación se presentan aspectos relevantes extraídos del instrumento de recolección. En primera instancia se indagó sobre, el nivel de formación de los empresarios (Cuadro 4), encontrándose que la mayor parte de los gerentes $(36,4 \%)$ poseen formación de bachilleres, seguido de los ejecutivos con formación de pregrado con un 27,7 $\%$ y en tercer lugar los gerentes con formación de técnicos con un $15,5 \%$, hecho que indiscutiblemente demuestra la baja formación profesional de los gerentes de estas empresas.

\begin{tabular}{|c|c|c|}
\hline Tipo de formación & Frecuencia & Porcentaje \\
\hline $\begin{array}{c}\text { No } \\
\text { Primaria } \\
\text { Bachiller } \\
\text { Técnico } \\
\text { Tecnólogo } \\
\text { Profesional } \\
\text { Especialización } \\
\text { Maestría } \\
\text { Total }\end{array}$ & $\begin{array}{c}8 \\
16 \\
129 \\
55 \\
22 \\
98 \\
20 \\
6 \\
354\end{array}$ & $\begin{array}{c}2,3 \\
4,5 \\
36,4 \\
15,5 \\
6,2 \\
27,7 \\
5,6 \\
1,7 \\
100,0\end{array}$ \\
\hline
\end{tabular}

Igualmente se encontró como los niveles de formación avanzada son los que menos se presentan, puesto que los gerentes con formación de maestría no superan el $2 \%$ y los de formación de especialistas tan solo alcanzan el 5,6\% del total de ejecutivos.

Igualmente, se indagó en torno al tema de las capacitaciones recibidas por los gerentes (Gráfica 3),

\section{Gráfica 3. Uso de los servicios de un centro de consultoría y asesoría empresarial}

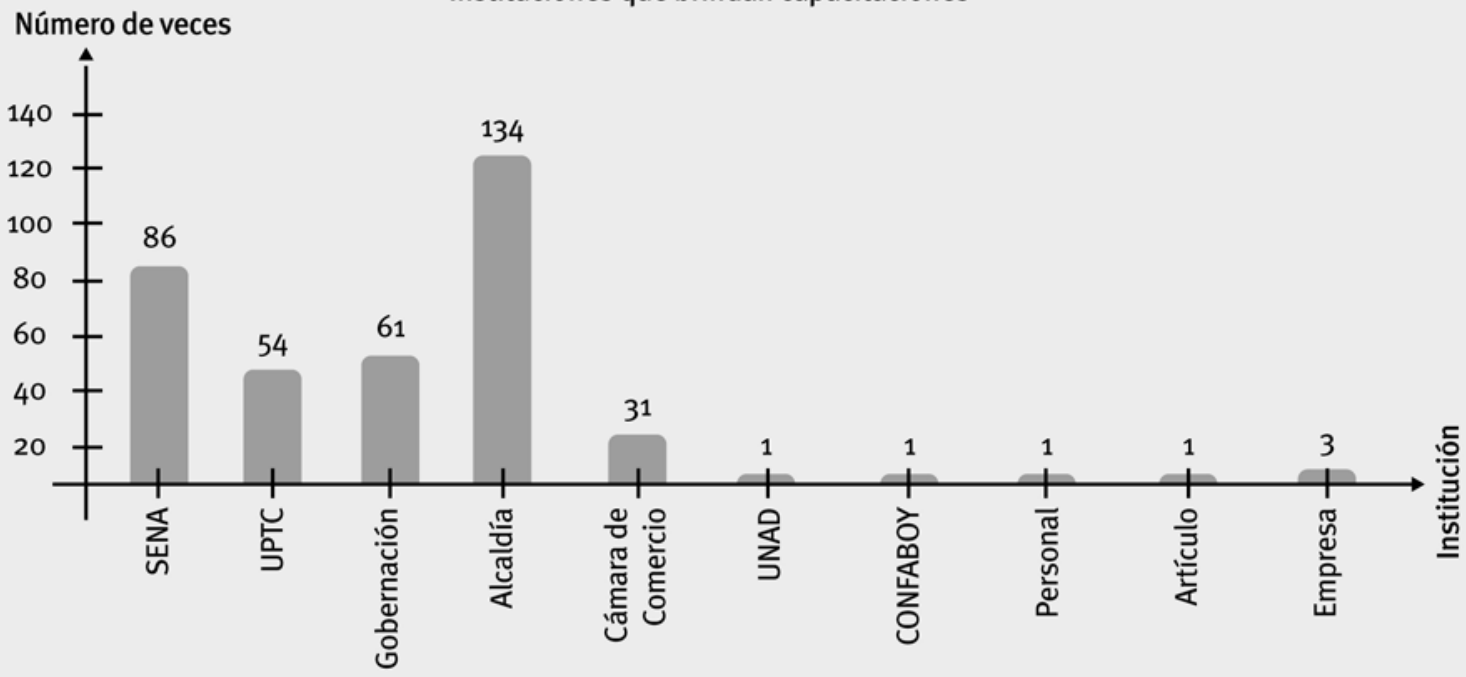


en torno a lo cual se encontró que son las Alcaldías las entidades que mayor cantidad de capacitaciones proporcionan a los gerentes (134 veces), seguidas del SENA con un total de 86 capacitaciones, en tercer lugar aparece la gobernación del departamento de Boyacá con 61 veces, seguidas de la Universidad Pedagógica y Tecnológica de Colombia - UPTC con 54 ocasiones y en quinto lugar la Cámara de Comercio de Sogamoso con 31 eventos.

Así mismo, se indagó sobre la disponibilidad, por parte de los empresarios, para el uso de los servicios del Centro de Consultoría y Asesoría Empresarial y el porqué de su utilización; los gerentes respondieron en un margen del $99 \%$ que las empresas de la ciudad de Sogamoso estarían dispuestas a contratar los servicios del Centro de Consultoría y Asesoría de la Universidad Pedagógica y Tecnológica de Colombia (UPTC), sede Sogamoso y el $1 \%$ de las empresas no utilizarían dichos servicios (Gráfica 4).

\section{Gráfica 4. Uso de los servicios de un centro de} consultoría y asesoria empresarial

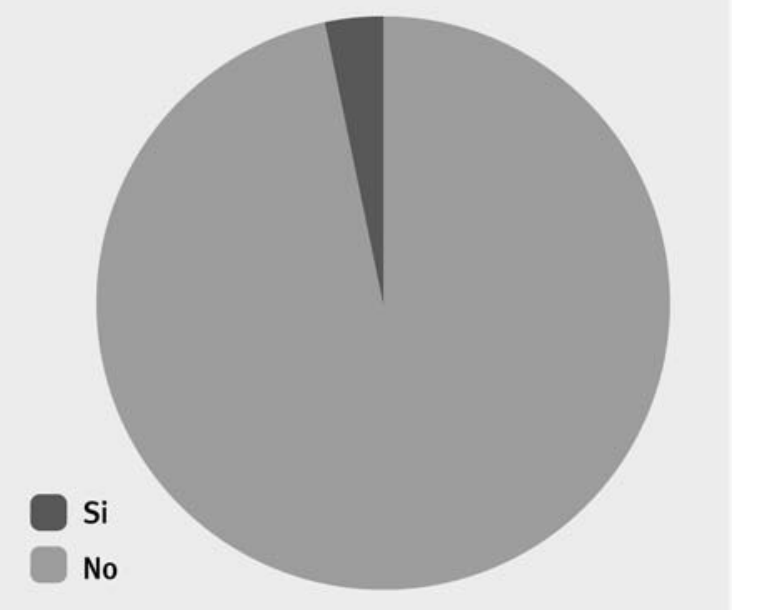

Fuente: elaboración propia.
Ahora, en cuanto a las razones de uso del servicio, muchas de las respuestas confirman la necesidad de crear el Centro de Consultoria y Asesoria Empresarial, ya que la población encuestada afirma que, en algunas ocasiones, no saben cómo resolver los diversos problemas que se presentan en sus empresas; así mismo, la asesoria y consultoría sería una oportunidad para mejorar la calidad del servicio o productos que ofrecen.

En cuanto a las diferencias que, según los gerentes y empresarios tenían en torno del concepto de Asesor y Consultor (Tabla 1), el $44 \%$ de las empresas encuestadas entiende que Consultor es la persona que despeja inquietudes; el $28 \%$, opina que es la persona que resuelve fallas en la empresa, el $17 \%$, dice que es la persona que capacita a los miembros de la empresa en temas determinados y el $11 \%$, contesto que es la persona que soluciona los problemas de la empresa (Tabla 1)

El $55 \%$ de las empresas encuestadas entienden que el Asesor es quien analiza información; para el $38 \%$ asesor es la persona que ofrece información para solucionar problemas y el $7 \%$ opina que es la persona que ayuda a las empresas a tomar decisiones. En consecuencia, las empresas encuestadas no tienen bien definido el concepto de Asesor ni de consultor, hecho que sería, para el cliente, un inconveniente en el momento de solicitar los servicios porque no tendría claro cuál de estos servicios requiere.

En cuanto al tipo de apoyo que más se solicitaría, se encontró que el $48 \%$ solicitaría el servicio de Asesoría, el $29 \%$ el de Consultoría y el $23 \%$ solicitaría ambos servicios. (Gráfica

\begin{tabular}{|c|c|c|c|}
\hline & Respuesta & Cantidad & Porcentaje \\
\hline Asesor & $\begin{array}{l}\text { Despeja inquietudes } \\
\text { Resuelve fallas de la empresa } \\
\text { Capacita } \\
\text { Soluciona problemas }\end{array}$ & $\begin{array}{l}156 \\
99 \\
60 \\
39\end{array}$ & $\begin{array}{l}44 \% \\
28 \% \\
17 \% \\
11 \%\end{array}$ \\
\hline Total & & 354 & $100 \%$ \\
\hline Consultor & $\begin{array}{l}\text { Despeja inquietudes } \\
\text { Ofrece información } \\
\text { Ayuda en la toma de decisiones }\end{array}$ & $\begin{array}{c}195 \\
134 \\
25\end{array}$ & $\begin{array}{c}55 \% \\
38 \% \\
7 \%\end{array}$ \\
\hline Total & & 354 & $100 \%$ \\
\hline
\end{tabular}


5). Se puede, concluir entonces, que la mayoría de empresas encuestadas se inclina por los servicios de Asesoría, puesto que considera que es un servicio mucho más económico. (Gráfica 5)

\section{Gráfica 5. Tipo de servicios de apoyo solicitados}

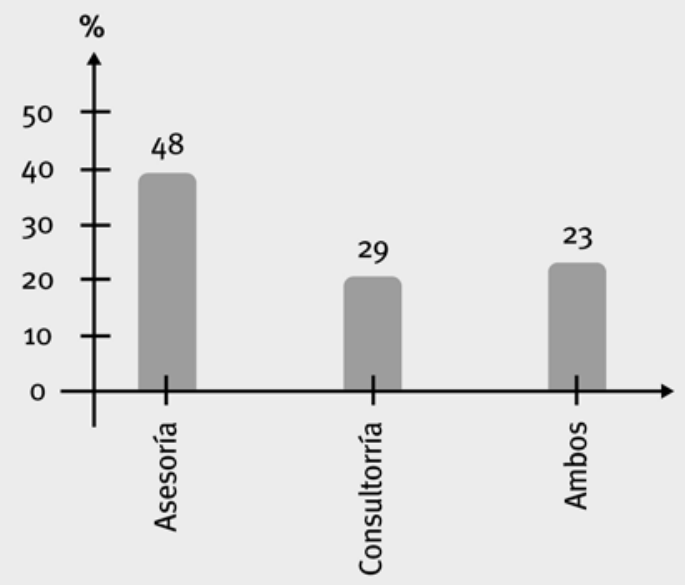

Fuente: elaboración propia.

Tabla 2. ¿La empresa cuenta con los recursos necesarios para contratar un Consultor o un Asesor Externo?

\begin{tabular}{|c|c|c|c|}
\hline & Respuesta & Cantidad & Porcentaje \\
\hline Asesor & SI & 78 & $22 \%$ \\
\hline Total & NO & 276 & $78 \%$ \\
\hline Consultor & SI & 354 & $100 \%$ \\
\hline Total & NO & 103 & $29 \%$ \\
\hline & & 354 & $71 \%$ \\
\hline & \multicolumn{3}{|c|}{ Fuente: elaboración propia. } \\
\hline
\end{tabular}

En el apartado relacionado con la exploración de los recursos necesarios para llevar a cabo un proceso de contratación seria de un servicio de consultoría o de asesoría a nivel externo, se encontró que, en su gran mayoría, los empresarios manifiestan no tener los recursos suficientes para este fin (Tabla 3 )

\section{Tabla 3. ¿Considera que el servicio de consultoría}

empresarial le puede generar mayor crecimiento

y desarrollo económico?

\begin{tabular}{|c|c|c|}
\hline Respuesta & Cantidad & Porcentaje \\
\hline Si & 308 & $87 \%$ \\
\hline No & 46 & $13 \%$ \\
\hline Total & 354 & $100 \%$ \\
\hline
\end{tabular}

Fuente: elaboración propia.

En cuanto a los recursos necesarios para la contratación de servicios de un Consultor, el $78 \%$, no cuenta con ellos; el $22 \%$ contesto que sí cuenta con los recursos; Ahora bien, de las 354 empresas, el $71 \%$, manifiesta no poseer recursos para contratar un Asesor; dado que, según lo expresaron los gerentes los precios actuales del mercado son muy altos. En tanto que, tan sólo un $29 \%$ sí cuenta con disponibilidad presupuestal para este fin. Ante interrogante sobre la disponibilidad de recursos que permita, a las compañías tener acceso a los servicios de consultoría, las respuestas tendieron a buscar tarifas cómodas y bajas, como lo muestra la (Gráfica 6)

\section{Gráfica 6. ¿Cuánto pagaría por la Consultoría} Empresarial?

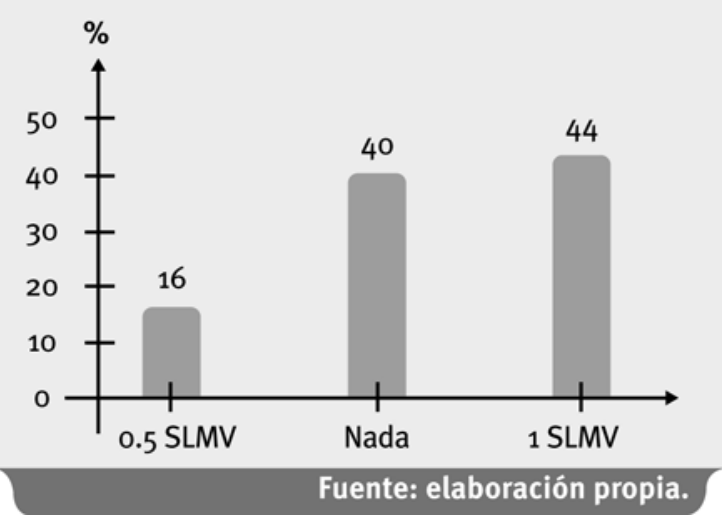

La gráfica permite apreciar que el $40 \%$ de las empresas encuestadas no pagaría nada por la prestación del servicio de Consultoría Empresarial porque son microempresas que no cuentan con los recursos económicos para acarrear estos costos; el $16 \%$ estaría en disposición de pagar hasta 0.5 Salarios mínimos legales vigentes (SMLV) y el $44 \%$ pagaría hasta un salario mínimo legal vigente. Se puede observar entonces, que existe un gran porcentaje del mercado por captar aunque dentro del estudio de demanda se refleja claramente que la población objeto de estudio tiene poca cultura de contratación de estos servicios, la tendencia es más al bajo precio y a la gratuidad, antes que al pago justo.

También se cuestionó a los gerentes sobre la visión prospectiva del servicio de Consultoría Empresarial y la posibilidad de tener mayor crecimiento y desarrollo económico en la región, aspecto que fue contundente en el sentido de ratificar en un alto porcentaje, las bondades del servicio. (Tabla 3 )

La tabla muestra que del $100 \%$ de las empresas encuestadas, el $87 \%$ sí cree que con el servicio de Consultoría Empresarial se tendría un mayor crecimiento y desarrollo económico en lo regional, puesto que se puede contar con 
personas capacitadas en el mercado; por otro lado, tan solo un $13 \%$, no cree que el servicio de Consultoría pueda contribuir a un mayor crecimiento y desarrollo económico en la región.

Otro de los interrogantes fue sobre las áreas con mayores dificultades que, según los Gerentes, deberían priorizarse para el servicio de consultoría. La Gráfica 7 permite apreciar que el $29 \%$ de las empresas presentan mayores problemas en el área de ventas; el $22 \%$, en el área de mercadeo, el $15 \%$, en administración, el $12 \%$, en contabilidad, el $8 \%$ en atención al público, el $8 \%$, en productividad y el $5 \%$ de las empresas encuestadas no contestaron. Lo anterior define claramente, que las empresas tienen inconvenientes en todas sus áreas y, por

\section{Gráfica 7. ¿En qué áreas de su empresa se presenta} mayores problemas?

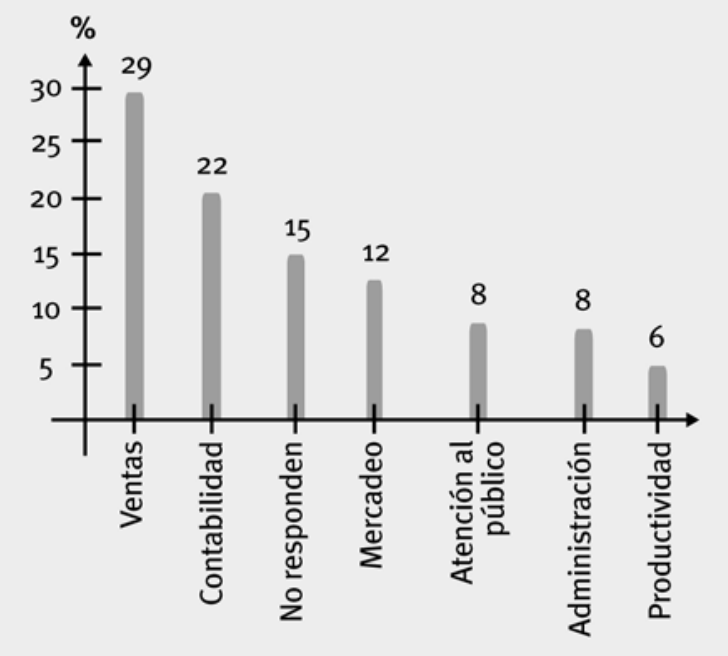

Fuente: elaboración propia.

lo tanto, es necesario contar con los servicios de Consultoría Empresarial para poder desarrollar estrategias que permitan solucionar estas dificultades.

Un aspecto relevante tiene que ver con los consultores internos; al respecto se cuestionó si las entidades contaban con consultores de la organización para solucionar los diferentes inconvenientes de la empresa. El $79 \%$ de las empresas encuestadas manifestó no contar con un Consultor interno que analice las deficiencias y fortalezas de la empresa para crear alternativas de solución a cada uno de los problemas que se presentan en las diferentes áreas de la empresa y, tan solo, un $11 \%$ afirma poseer estos servicios; el $10 \%$ no respondio esta pregunta. (Tabla 4)
Tabla 4. ¿Su empresa cuenta con Consultores

internos para ayudar a la solución de problemas que se presentan en la empresa?

\begin{tabular}{|c|c|c|}
\hline Respuesta & Cantidad & Porcentaje \\
\hline Si & 39 & $11 \%$ \\
\hline No & 280 & $79 \%$ \\
\hline No responden & 35 & $10 \%$ \\
\hline TOTAL & 354 & $100 \%$ \\
\hline \multicolumn{2}{|c|}{ Fuente: elaboración propia. } \\
\hline
\end{tabular}

Por último, se indagó sobre las distintas opiniones y sugerencias para la creación de un Centro de Consultoría y Asesoría Empresarial en Sogamoso (para la provincia de Sugamuxi). Como lo evidencia la gráfica 8 el $28 \%$, opina que el servicio de Consultoría y Asesoría debe ser gratuito; el $19 \%$ manifiesta que el servicio debe desarrollar tareas con mucha responsabilidad, el $18 \%$ afirma que la capacitación sea clara y precisa. Además el $12 \%$ consideró que el servicio debe estar al alcance de todas las empresas; el $8 \%$ habló acerca de la eficacia del servicio y el $7 \%$ que el servicio de Consultoría y Asesoría Empresarial debe poseer creatividad a la hora de fijar estrategias de servicio (Gráfica 8)

Gráfica 8. Opiniones y sugerencias para la creación del

Centro de Consultoríay Asesoría de la UPTC Sede Sogamoso

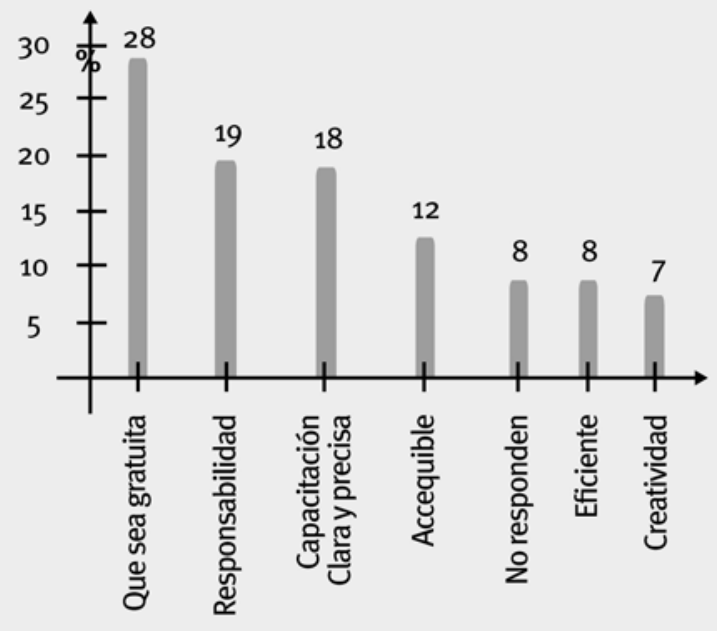

Fuente: elaboración propia.

\section{Conclusiones}

La puesta en escena de este trabajo investigativo serio y riguroso permitió identificar una serie de dificultades, relacionadas con las $\mathrm{Mi}$ pymes en la ciudad de Sogamoso. La encuesta, como instrumento generador de información, se convirtió en la piedra angular, mediante la cual se pudo comprobar que el $99 \%$ de las empresas 
de esta región están en disposición de utilizar los servicios de consultoría, siempre y cuando se tenga claridad en cuanto a los conceptos y funciones que realiza un Consultor y/o un Asesor en las empresas, puesto que gran parte de la población encuestada ( $48 \%$ ) requiere los servicios de un Asesor; para el crecimiento y desarrollo de la empresa.

La mayoría de la población objeto de estudio (70\%), no cuenta con recursos económicos necesarios para contratar los servicios de Consultoría Empresarial; sin embargo, ve la necesidad apremiante de buscar ayuda para mejorar la calidad de sus servicios o productos y, así poder aumentar su competitividad en el mercado. A la vez el $55 \%$ de las empresas no están en disposición económica para contratar dichos paquetes de servicios, precisamente porque son microempresas que no cuentan con los recursos necesarios para sumir estos gastos. Es necesario concluir que la falta de Consultoría hace que muchas de las empresas sufran estancamiento en su producción y desaprovechen la posibilidad de crecimiento y mejoramiento continuo para ser competitivas en el mercado actual.

Otro de los resultados relevantes en la investigación se relaciona con las áreas que revisten mayores problemas. El dato obtenido, según las empresas encuestadas es: el área de Ventas (29\%); el área de Mercadeo (22\%); la parte Administrativa el ( $15 \%)$; lo anterior se debe a que las empresas no cuentan con un Consultor que analice las deficiencias y fortalezas de la empresa, para crear alternativas de solución en cada uno de los problemas evidenciados.

Se enfatizó sobre la importancia de prestar atención a esta población empresarial, mediante la solución de problemas que se presentan en sus empresas; pues, en el momento, únicamente se están presentando alternativas esporádicas que no permiten hacer un estudio concienzudo sobre la situación; por tanto, no se presentan estrategias que prevengan y corrijan el problema encontrado de forma efectiva y precisa.

En suma, en cuanto a disposición económica para contratar servicios de consultoría empresarial, el $28 \%$ de las empresas encuestadas opinan que los servicios deben ser gratuitos, mediante la creación de vínculos entre empresa, universidad, futuros profesionales prestadores de los servicios de asesoría y la misma institución educativa, para el caso la UPTC, sede
Sogamoso, ciertamente mediante parámetros de responsabilidad y cumplimiento de las tareas por desarrollar.

De la misma manera se encontró que en lo referente al nivel de formación empresarial, es la media la que se impone con un $36,4 \%$ de gerentes bachilleres, seguida de los ejecutivos con formación de pregrado con un $27,7 \%$, encontrándose en tercer lugar a un 15,5\% de directores con formación técnica, igualmente dicho interrogante permitió establecer que los niveles que menos se presentan son el doctorado con o\%, el de Maestría con 1,7\% y especialización con $5,6 \%$. Ante esta realidad se puede inferir que muchas de las deficiencias en materia de necesidades de consultoría y asesoría se pueden deber al desconocimiento y al bajo nivel de formación de los encargados de las compañías.

Por último se destacan también los procesos de educación y capacitación, aspecto en el cual se encontró que las entidades que mayor apoyo prestan a las Mipymes son en su orden las Alcaldías (134 capacitaciones), en segundo lugar el SENA (86), Gobernación (61), UPTC(54) y Cámara de Comercio con 31 capacitaciones, aspecto que demuestra una importante falencia en el tema debido al bajo número en promedio de dichas actividades de capacitación.

\section{Referencias}

Andrade, A. (2007). Consultores e consultoría: o que frazer para essa relação dar certo. REEAD, 1 (1). Extraído el 30 de abril de 2011 desde http://www.lasallerj.org/ revistas/index.php/administracao/article/ view/22/26.

Antonorsi, M. (2010). Consultoría interna: asesorarse con los nuestros: Debates IESA, 15(3), pp. 48-52.

Bezerra, A. (2002). Estudo sobre o impacto das consultorias Patme/Cin sobre a productividade das MPE'S. Tesis de Maestría. Programa de Posgraduados en Ingeniera de la producción. Universidade Federal de Pernanbuco. Extraído el 2 de junio de 2011 desde http://www.liber.ufpe.br/teses/ arquivo/20030718123711.pdf.

Block, P. (1991). Consultoría: un desafío de libertad. Sao Paulo, Brasil: McGraw- Hill. 
Bustelo, C. y García, E. (2000). Consultoría en la organización de la información. Profesional de la Información, 9(9), pp. 4-10.

Calderón G. y Castaño G. (2005). Investigación en administración en América latina evolución y resultados. Manizales, Colombia: Universidad Nacional.

Calegari, A. y Karyna, S. (2001). Consultoria e o profissional da informa consultoria del profesional de la informação: um campo em expansão. $A C B$ : Bibliotecomania em Santa Catarina, 6(1), pp. 146156.

Camacho, B., (2003). Metodología de la Investigación científica, un camino fácil de recorrer para todos. Tunja, Colombia: Universidad Pedagógica y Tecnológica de Colombia.

De Padua, A. y Duarte T. (2005). Significações culturais: um estudio de caso da UFMG consultoria junior. Revista de Administração Mackenzie, 6(3), pp. 137-166.

Donadone, J. (2003). 0 mercado de consultoria nas ùltimas décadas: crescimento, diversificação e formas de disputa. Cadernos de Pequisas em administração, 10(2), pp. 1-15.

Donina, A. (2009). Estudando um modelo de programa de mentoria formal brasileiro: Estudio de caso na empresa DELOITTE TOUCHE TOHMATSU. Tesis do Mestrado em administração. Recife, Brasil: Centro de pesquisa e posgraduação em administração - CPPA.

Duarte, L. y Fernández, A. (2006). Propuesta para la creación de un centro de consultoría y asesoría empresarial en la escuela de administración de empresas de la Universidad Pedagógica y Tecnológica de Colombia Sede Sogamoso. Tesis no publicada. Facultad de Ciencias Económicas y Administrativas, Universidad Tecnólogica de Colombia, Sede Sogamoso.

Fowler, J. y O'Gorman, J. (2005). Mentoring Functions: A contemporany view of the perceptions of Mentees and mentors. British journal of Management, n. 16, pp. 40 - 60 .

Gerusa, A. (2002). 0 papel do consultor administrativo externo no fortalecimento do negocio. Tesis do grau de Bacharel em Administração. Universidad Federal do Pará. Extraido el 15 de mayo de 2011 desde http://www.planoaudito- ria.com.br/site/download/110908_monografia_adna.pdf.

Greiner, L. y Metzger, R. (1983). Consulting to management. Englewood cliffs, NJ: Prentice- Hall.

Hernández, J. (2003). El placer de ser consultor. E-Semanal, 21(577), pp. 1-5.

Hompanera, F. (2002). ¿La consultoría es cara?. E-Semanal, 21(521), pp. 24-26.

INSTITUTO DE CONSULTORES DE EMPRESAS DE MEXICO. (2010). Concepto de Consultoría. Extraído el 15 de julio de 2011 desde http://www.mitecnologico.com/Main/ConceptoDeConsultoria.

Jacintho, P. (2004). Consultoría empresarial: procedimientos para la aplicación en micro y pequeñas empresas. Tesis de Maestría. Florianopolis, Brasil: Programa de graduados de Ingeniera de la Producción, Universidad Federal de Santa Catarina.

Kubr, M. (1986). Consultoria: um guía para a profissão. Rio de Janeiro, Brasil: Guanabara.

Kubr, M. (2007). La consultoría de Empresas. México D.F.: Limusa.

López, E., Sepúlveda, C.y Arenas, H. (2010). La consultoría de Gestión Humana en empresas medianas. Estudios Gerenciales, 26(114), pp. 149-168.

Lozano, M. (2009). Elementos para la consultoría en empresas familiares. Pensamiento y Gestión, n. 26, pp. 214-237.

Mafra, F. y Rodrigues, R. (2007). Uso de fontes de informação por consultores empresariais: um estudo junto ao mercado de consultoria de Belo Horizonte. Perspectivas em ciência da informação, 13(1), pp. 95-111.

Marc, P. y Stuart, M. (1994). Sea su propio consultor y ahorre dinero: guía para desarrollar una consultoría interna eficaz. Barcelona, España: Folio.

Méndez, C. (1998). Metodología, Guía para Elaborar Diseños de Investigación en Ciencias Económicas, Administrativas y contables, Fundamentos Teóricos. Bogotá, Colombia: Mc Graw Hill.

Mictecnológico (2010). Concepto de Consultoría. Extraído el 3 de junio de 2011 desde www.mitecnologico.com/Main/ConceptoDeConsultoria. 
Miranda A. y Vitta J. (2008). La consultoría organizacional o de procesos. Trabajo presentado en el XXX Simposio Nacional de Profesores de Práctica Profesional. Universidad Nacional del Rosario, Rosario-Argentina, Ocubre. Extraído el 3 de junio de 2011 desde http://www.econ. uba.ar/www/institutos/contable/ceconta/ Foro_practica_profesional/Simposios\%20Practica\%2oPDF/Rosario/Metodolog\%C3\%ADa/ SimposioXXX_M_04_Rocha_Vitta.pdf.

Moreno, J. (2006) ¿Cómo la consultoría puede generar valor? E-Semanal, pp. 1-4.

Neves, A. y Campello, B. (2008). Analisando o impacto da consultoria interna no sector público. Economia \& Gestão da PUC Minas, 8, (18), pp. 53 $-71$.

Orlickas, E. (1998). Consultoría interna de recursos humanos: conceptos, casos y estrategias. Sao Paulo, Brasil: Makron Books.

Parreira, F. (1997). Consultoría: consultores y Clientes. Sao Paulo, Brasil: Erica.

Pequeña y Mediana Empresa. (2010). Conoce el reporte que ayudará a tu PYME a sobrevivir a esta crisis. Extraído el 4 de mayo de 2011 desde www. pyme.com.mx/administracion/index.php.

Pérez, A. (2006). Mas demanda em consultoria de Valor. E-Semanal, n. 691, pp. 23-26.
Piovezan, L. (2008). Consultoria em pequena empresa do setor de instalações: viabilidade do uso do método da pesquisa-ação. Revista Eletrônica de Educação e Tecnologia do SENAI-SP, 2(3), pp. 1-32.

Ribeiro, F.y Ribeiro M. (2010). 0 espíritu do acordó em uma consultoria de planejamiento estratégico. Extraído el 4 de mayo de 2011 desde http://www. ead.fea.usp.br/eadonline/grupodepesquisa/ publica\% ${ }_{3} \% \mathrm{~A}_{7} \% \mathrm{C}_{3} \% \mathrm{~B}_{5} \mathrm{es} / \mathrm{martinho} / 46$. pdf.

Schein, E. (1988). Consultoría de procesos, su papel en el desarrollo organizacional. Wilmington, Delaware, EUA: Addison - wesley Iberoamericana, Fondo Educativo Interamericano, S.A.

Tamayo, M. (2002). El Proceso de la Investigación Científica, La Investigación Científica. Balderas. Mexico D.F.: Limusa Noriega Editores.

Taylor, F. (1911). The principies of scientific management. New York: Harper and Brothers.

Universidad Pedagógica y Tecnológica de Colombia. (2010). Informe del cuatrienio 2007-2010. Extraído el 4 de mayo de 2011 desde www.uptc.edu.co/.

Velásquez, F.y Vernaza, P. (2006). IBM: más allá del conocimiento? Estudios Gerenciales, 22(101), pp. 125-148.

Viadu, F., Marimon, F., Casadesús, M., Heras, I. (2006). Consultoría en España. Universia Business Review, n. 9, pp. 74-87. 